\title{
Inductively Coupled Plasma-Mass
}

Spectrometry Analysis of Plutonium Samples

\section{Los Alamos}


An Affirmative Action/Equal Opportunity Employer

This report was prepared as an account of work sponsored by an agency of the United States Government. Neither The Regents of the University of California, the United States

Government nor any agency thereof, nor any of their employees, makes any warranty, express or implied, or assumes any legal liability or responsibility for the accuracy, completeness, or usefulness of any information, apparatus, product, or process disclosed, or represents that its use would not infringe privately owned rights. Reference herein to any specific commercial product, process, or service by trade name, trademark, manufacturer, or otherwise, does not necessarily constitute or imply its endorsement, recommendation, or favoring by The Regents of the University of California, the United States Government, or any agency thereof. The views and opinions of authors expressed herein do not necessarily state or reflect those of The Regents of the University of California, the United States Government, or any agency thereof. Los Alamos National Laboratory strongly supports academic freedom and a researcher's right to publish; as an institution, however, the Laboratory does not endorse the viewpoint of a publication or guarantee its technical correctness. 


\section{Inductively Coupled Plasma-Mass}

Spectrometry Analysis of Plutonium Samples

\section{Deborah Figg}

Alex Martinez

Lawrence Drake

Chris Brink 


\title{
Inductively Coupled Plasma-Mass Spectrometry Analysis of Plutonium Samples
}

\author{
Deborah Figg, Alex Martinez, Lawrence Drake, and Chris Brink
}

\begin{abstract}
At Los Alamos National Laboratory (LANL), Inductively Coupled Plasma-Mass Spectrometry (ICP-MS) is routinely used to analyze a wide variety of plutonium samples for inorganic analytes. Approximately $75 \%$ of the elements of the periodic table can easily be determined by ICP-MS analysis. Techniques that use atomic emission for element detection and quantification encounter a large number of spectral interferences when analyzing plutonium samples due to the numerous emission lines of plutonium. Mass spectrometric techniques do not have that problem. In ICP-MS the main effect plutonium has on the elemental determinations is a signal suppression. With matrix dilution and signal normalization that problem is mitigated. This report describes the ICP-MS method used in the analysis of plutonium samples and the resulting detection limits.
\end{abstract}

\section{Introduction}

Presently, LANL has 2 glovebox ICP-MS instruments for the analysis of trace elements in radioactive samples. These instruments are housed in the Chemistry and Materials Research building (CMR) and are operated by NMT-1, the analytical chemistry group in the Nuclear Materials Technology (NMT) division. One of the instruments was used for 4-5 years to analyze trace actinides in brine solution in support of the Source Term Waste Test Program (STTP) project for the Waste Isolation Pilot Plant (WIPP). That instrument is now being used for trace elements in actinide materials, as is the second instrument. The samples analyzed are in support of Pit Rebuild, Pit Surveillance, Mixed Oxide Fuel (MOX), Pit Disassembly and Conversion, Materials Identification Survey (MIS), 238 Plutonium Heat Source, and waste stream analysis programs. In the past, we have also supported Hanford and Rocky Flats projects.

Inductively Coupled Plasma-Mass Spectrometry (ICP-MS) measures ions produced by a radio-frequency inductively coupled plasma. Analyte species originating in a liquid are nebulized and the resulting aerosol is transported by argon gas into the plasma torch. The ions produced are entrained in the plasma gas and introduced, by means of a watercooled interface, into a quadrupole mass spectrometer. The ions produced in the plasma are sorted according to their mass-to-charge ratios and quantified with a channel electron multiplier.

The typical routine sample requires an acid digestion with a combination of nitric, hydrochloric, and hydrofluoric acids. The digested sample is split into a portion for ICPMS analysis, and a portion for Inductively Coupled Plasma-Atomic Emission Spectroscopy (ICP-AES) analysis. Prior to the ICP-AES analysis, ion chromatography is used to remove the plutonium from the samples. In general, ICP-AES is used for the 
lighter elements ( $\mathrm{Na}, \mathrm{Ca}, \mathrm{K}, \mathrm{Si}$, first row transition metals, etc.) and ICP-MS for the heavier elements.

\section{Instrumentation}

The glovebox ICP-MS instruments (VG Elemental Franklin, MA, USA) utilize a quadrupole mass spectrometer that operates at 1 atomic mass unit (amu) resolution. The oldest instrument is a first generation PlasmaQuad 2 (PQ2). The second instrument is a PlasmaQuad PQ2-STE (PQS). Both instruments have been designed for glovebox operations. Only the necessary components of the instrument are inside the negative pressure glovebox. These components include the autosampler, torchbox, and a majority of the vacuum chamber. The instrument racks and vacuum pumps are outside the glovebox.

The PQ2 has undergone several manufacturer upgrades that have increased the analyte sensitivity and instrument reliability, ensured quality performance, and extended the instrument's normal lifetime. The tube style Henry ICP and quadrupole radio-frequency generators have been replaced with solid-state generators, the diffusion pumps have been replaced with turbo pumps, and, in addition, the interface region has had a 'modified enhanced interface' upgrade to produce higher sensitivity. This was not a standard VG Elemental 'enhanced interface upgrade' but did involve changing the expansion chamber vacuum pump and the spacing between the sampler and skimmer cones.

Typical ICP-MS instrument parameters are used during an analysis. The ICP generator is operated at a forward power of 1350 watts; a concentric nebulizer and a Scotts spray chamber are used; the coolant gas flow rate is $15 \mathrm{~L} / \mathrm{min}$., auxiliary gas flow rate is 1 $\mathrm{L} / \mathrm{min}$., and the nebulizer gas flow rate is $0.7 \mathrm{~L} / \mathrm{min}$.

\section{Reagents}

The reagents used for trace elemental analysis are concentrated ultra high-purity nitric acid (Fisher Scientific, OPTIMA or equivalent), $10 \mathrm{ppm}$ multielement or single element standards (SPEX, High Purity, or equivalent), high purity liquid argon (99.999\%), and laboratory accepted demineralized or deionized water $(18 \mathrm{M} \mathrm{ohm}-\mathrm{cm})$. One percent nitric acid is used as a rinse solution. The rinse solution is prepared by adding ten milliliters of concentrated ultra high-purity nitric acid to less than one liter of deionized water and diluting to one liter with water. Stock solutions $(10 \mathrm{ppm})$ of multielement standards are prepared from single element standards and stored in polytetrafluoroethylene (PTFE) bottles. These solutions are prepared in-house and have been found to be stable for one year. These solutions are used as calibration standards. A second set of commercially prepared $(10 \mathrm{ppm})$ multielement standards are used for initial calibration verification (ICV) and continuing calibration verification (CCV) standards during analytical runs. Disposable calibrated Falcon tubes, made of polypropylene, are used to prepare dilutions of samples for analytical runs. All dilutions of samples are done volumetrically. Rainin electronic pipettes are used to deliver the appropriate volume of sample for dilution. Balance and pipettes are checked each day to ensure proper operation. The acid concentration for the prepared dilutions is $1 \% \mathrm{HNO}_{3}$. 


\section{Interferences}

Unlike atomic emission techniques, ICP-MS does not suffer from the numerous spectral interferences resulting from the actinide matrix. The only ICP-MS actinide related spectral interferences observed are those from the actinide $2+$, actinide-O $2+$, and actinide- $\mathrm{H}$. When analyzing a plutonium-239 sample the interferences from the matrix are at $120.5,127.5$, and $240 \mathrm{amu}$. Typically a sample contains more than one actinide isotope that interferes. These interferences affect tin, tellurium, and antimony. Tin and tellurium are seldom requested. Antimony is determined when Resource Conservation and Recovery Act (RCRA) listed elements are requested and the 123 isotope is used. Since the instruments are used to analyze actinide matrix samples the actinide background is high, and trace actinide determinations cannot be made on these instruments. Therefore the actinide- $\mathrm{H}$ interference is of no concern.

ICP-MS does have spectral interferences that result from the atmospheric argon plasma and the acid solution used to introduce the sample into the plasma. These interferences are well documented in the literature. ${ }^{1,2}$ Basically there are varying degrees of spectral interferences from about 12 amu to $80 \mathrm{amu}$. About half of the elements with isotopes in this range can still be determined using a quadrupole-based ICP-MS instrument. Another technique has to be used for elements such as N, O, F, C, Si, S, Na, Cl, K, Ar, and low levels of $\mathrm{Fe}$ and $\mathrm{Ca}$. If hydrochloric acid is used as the solvent, arsenic (As) is difficult to analyze for with a quadrupole instrument.

The main effect plutonium has on the elemental determinations is a signal suppression that results in a decrease in sensitivity and poor analyte recoveries unless treated properly. Figure 1 shows the signal suppression that occurs in different concentrations of a plutonium matrix. This data was obtained using very pure plutonium metal. The plutonium metal was digested and then diluted to the 3 different concentrations shown in Figure 1: 1000,500 , and $100 \mu \mathrm{g} / \mathrm{ml}$. The 3 solutions were spiked with over 30 elements at 3-10 times their detection limit. Therefore most of the trace elements are spiked at the single to tens of $\mathrm{ng} / \mathrm{ml}$ (ppb) level. The signals for each of the spiked elements in the 500 and $1000 \mu \mathrm{g} / \mathrm{ml}$ solutions were normalized to their signal in the $100 \mu \mathrm{g} / \mathrm{ml}$ solution.

Figure 1 shows that as the plutonium concentration in the sample increases, the signal of all trace analytes decreases. This type of matrix-related signal suppression resulting from large concentrations of heavy ions has been documented. ${ }^{3-5}$ In this case the plutonium ion is causing the signal suppression. 
Figure 1: Signal Suppression for Several Elements as a Function of the Plutonium Concentration.

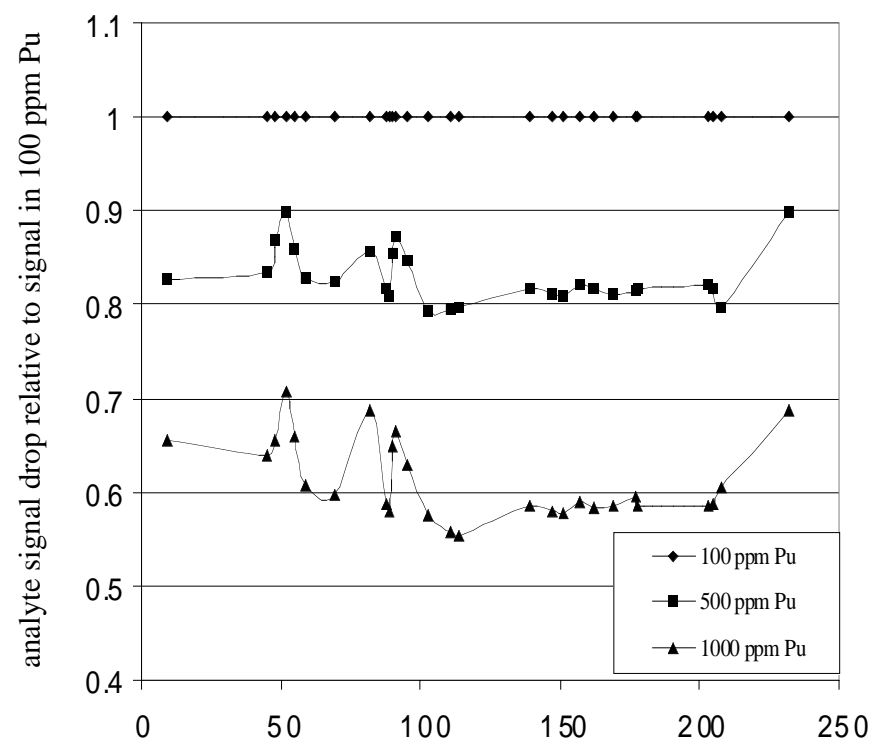

AMU of analyte

The signal suppression is relatively constant across the mass range. There are some elements that are not affected as much as the other elements. The biased results may be an artifact or a true signal enhancement of those signals relative to the other elements. There are many possible reasons this may be occurring, but at this time the exact reason is not known.

One method used to correct this type of suppression is through signal normalization. This is accomplished through the use of internal standards, which are used to monitor and correct for the signal suppression and other forms of drift that might occur during an analysis. Figure 2 shows the effectiveness of this correction for elements in different concentrations of plutonium solutions. The data in Figure 2 came from the same solutions used to produce Figure 1. Figure 1 shows what happens to the analyte signals. Figure 2 shows how well the calculated concentration of each spiked analyte compares to its true value. The calculated value is determined by first normalizing the signal with the internal standard, and then using a calibration curve to convert the normalized signal to a concentration. The calibration curve for each analyte is determined using elemental standards in $2 \% \mathrm{HNO}_{3}$ solutions. As shown in Figure 2, the calculated values for the trace analytes are recovered well at lower plutonium concentrations, but not at higher plutonium concentrations. For this reason, plutonium samples are diluted to concentrations of $500 \mu \mathrm{g} / \mathrm{ml}$ or less prior to analysis. 
Figure 2: \% Recovery [100 $\times($ calculated value / true value $)]$ of the Spiked Trace Analytes in Different Plutonium Concentrations.

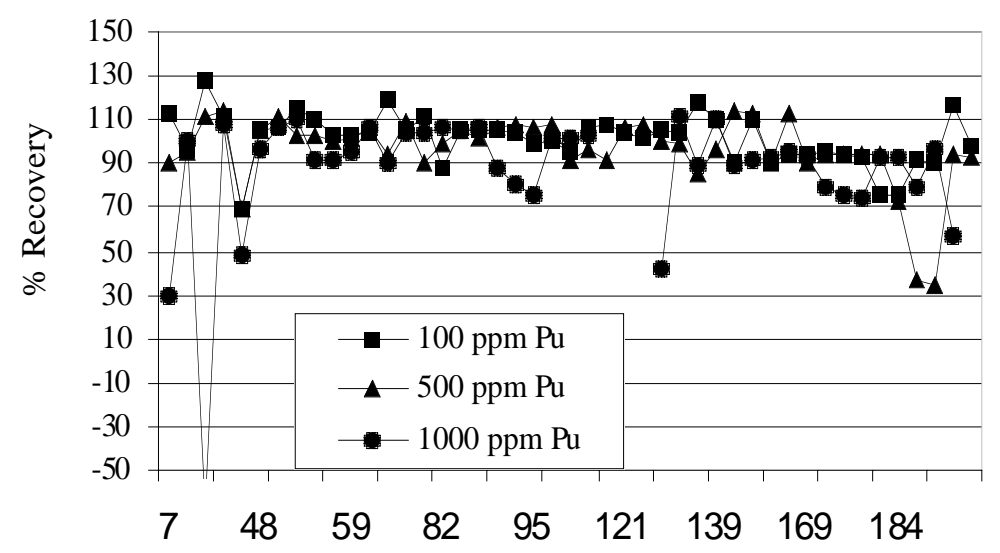

\begin{abstract}
AMU of element
Because the instrument response decreases with increasing plutonium matrix, the limits of the detection increase with increasing plutonium matrix. Even though the detection limits at the instrument are better in more dilute samples, the result of further sample dilution is a higher dilution factor. The dilution factor is used to convert the value obtained at the instrument to the corresponding value in the original solid sample. The higher the dilution factor the higher the multiplier. So the final method used has to balance the effects of signal suppression and how well the internal standards correct for it, and the detection limits required by the customer. A routine plutonium metal or oxide sample is typically diluted to 500 and $10 \mu \mathrm{g}$ sample $/ \mathrm{ml}$. The $500 \mu \mathrm{g} / \mathrm{ml}$ sample concentration routinely produces final detection limits required by our customers. Typically, the further dilution of the sample to $10 \mathrm{ppm}$ does not give detection limit values that meets the customers requirements, but it is used to quantify analytes that are present in the solid sample at levels greater than 200 to $300 \mathrm{ppm}$.

\section{Analysis Method}

Prior to ICP-MS analysis the plutonium samples must first be digested with strong acid solutions. Plutonium metals are easily digested with $6 \mathrm{M} \mathrm{HCl}$. Oxide and miscellaneous plutonium and uranium materials are digested in a microwave, hot block, or sealed reflux tube. The microwave technique starts with $12 \mathrm{M}$ nitric acid $\left(\mathrm{HNO}_{3}\right)$ and $0.1 \mathrm{M}$ hydrofluoric acid (HF). The sealed reflux digestion uses concentrated $\mathrm{HCl}$, and trace $\mathrm{HNO}_{3}$ and $\mathrm{HF}$. The digested sample is brought to a prescribed volume with deionized $\mathrm{H}_{2} \mathrm{O}$. ICP-MS analysts typically receive digested samples that have a sample concentration between $5000-10,000 \mu \mathrm{g} / \mathrm{ml}$. The sample cannot be analyzed at this concentration due to the matrix effects discussed in section IV.
\end{abstract}

To facilitate the analysis, the digested sample is diluted to a final volume of $5 \mathrm{~mL}$ with $1 \% \mathrm{HNO}_{3}$. Two dilutions from this sample are then analyzed. The first dilution is a 1:20 
dilution that reduces the plutonium concentration to less than $500 \mu \mathrm{g} / \mathrm{ml}$, minimizing any matrix effects. The 1:20 dilution also ensures that the customers' detection limit requirement can be met for analytes of low concentration. The 1:200 dilutions are used to quantify analytes that exceed the linear calibration range in the initial dilution. The internal standards used to monitor instrument drift are scandium (Sc), rhodium ( $\mathrm{Rh})$, and thulium (Tm). These internal standards are added to each sample in an analytical run at a concentration of $100 \mathrm{ppb}$.

A typical ICP-MS analysis run is described in Table 1. The analysis method incorporates quality control checks that monitor both the sample preparation process as well as instrument performance. A calibration blank (CBL) is used for blank subtraction. The calibration blank consists of the internal standards and the same kind and concentration of acid used to prepare the sample dilutions $\left(1 \% \mathrm{HNO}_{3}\right)$. Two calibration standards are processed with each analytical run. The low calibration standard (CSM) is prepared at a concentration of $20 \mathrm{ppb}$ and the high calibration standard (CSH) is prepared at a concentration of $100 \mathrm{ppb}$. Calibration check standards, which are prepared from a secondary source, are used to verify the calibration generated from the calibration standards. An ICV is run immediately after the CSH followed by an initial calibration blank (ICB). Calibration verification standards (CCVs) are run approximately every ten samples. Calibration verification standards are spiked at the midpoint of the linear calibration range. Reagent blanks and process blanks are used to monitor possible analyte contamination that may occur during sample digestion and processing of the digested sample. Analytes are spiked before and after digestion into 5-10\% of the samples to track analyte recovery during sample preparation and instrument analysis. Duplicate analysis or serial dilution is performed at the customer's request.

Table 1. Composition of a Typical Analytical Run

\begin{tabular}{|l|l|}
\hline CBL & Calibration blank \\
CSM & Calibration standard low \\
CSH & Calibration standard high \\
ICV & Initial calibration verification \\
ICB & Initial calibration blank \\
\hline \multicolumn{2}{|c|}{ 10/20 samples* } \\
\hline CCV & Continuing calibration verification \\
CCB & Continuing calibration blank \\
\hline \multicolumn{2}{|c|}{ 10/20 samples* } \\
\hline CCV & Continuing calibration verification \\
CCB & Continuing calibration blank \\
\hline
\end{tabular}

* "Samples" includes all the samples in an analytical batch, including the process blank, the matrix spike, the matrix spike duplicate and the post-digestion spike. Several analytical batches can comprise an analytical run. For the PQ2 ICP-MS instrument, an analytical run is limited to forty-four prepared solutions. 


\section{Detection Limits}

There are two detection limits that are used. One is the instrument detection limit (IDL) that is determined using blank solutions and the other is that which relates to the detection limit of an analyte in a sample. The instrument detection limits (IDL) are determined every six months or whenever a significant change to the instrumentation has occurred (e.g. replacement of a detector or quadrupole). The IDL is determined by multiplying by 3 the average of the standard deviations obtained for seven consecutive measurements of the blank solution on three nonconsecutive days from the analysis of an analyte free blank solution. The measurements are performed as though each blank were a separate analytical sample. That is, each sample is made individually in its own tube and a rinse is used after the measurement of each blank. The IDLs are determined for the instrumental configuration used in the analysis of the samples. The detection limit of an analyte in the sample is determined by multiplying the IDL by the dilution factor. The detection limits determined in this manner depend upon the dilution of the sample and the acid used, but do not depend upon the matrix. Typical IDLs and the limit of detection for the analytes in a solid sample are shown in Table 2 . It should be noted that the detection limits reported in Table 2 are derived under the conditions used in a typical multielement analysis. These detection limits can be improved under certain conditions and the customers' request, such as the case for phosphorous analysis.

Table 2. Current Detection Limits of each Glovebox ICP-MS for Analytes in Solution and Corrected Back to the Solid

(The dilution corrected values assume $20 \mathrm{mg}$ of digested sample are diluted to 5 $\mathrm{ml}$, then further diluted 1:20.)

\begin{tabular}{|l|c|c|c|c|c|}
\hline Element & Isotope & \multicolumn{2}{|c|}{ Detection Limits PQ2 } & \multicolumn{2}{c|}{ Detection Limits PQS } \\
\hline & & $\mathrm{ppb}(\mathrm{ng} / \mathrm{ml})$ & $\mathrm{ppm}(\boldsymbol{\mu g} / \mathbf{g})$ & $\mathrm{ppb}(\mathrm{ng} / \mathrm{ml})$ & $\mathrm{ppm}(\boldsymbol{\mu g} / \mathbf{g})$ \\
\cline { 2 - 6 } Beryllium & $\mathrm{Be} 9$ & 0.06 & 0.3 & 0.06 & 0.3 \\
Boron & $\mathrm{B} 11$ & 0.27 & 1.34 & 2.84 & 14.22 \\
Phosphorus & $\mathrm{P} 31$ & 2.44 & 12.20 & 5.76 & 28.82 \\
Titanium & Ti 49 & 0.44 & 2.22 & 1.31 & 6.54 \\
Vanadium & $\mathrm{V} 51$ & 0.36 & 1.78 & 0.94 & 4.68 \\
Chromium & $\mathrm{Cr} 52$ & 0.06 & 0.31 & 0.31 & 1.53 \\
Manganese & Mn 55 & 0.03 & 0.17 & 0.21 & 1.03 \\
Cobalt & Co 59 & 0.03 & 0.16 & 0.19 & 0.97 \\
Nickel & $\mathrm{Ni} 60$ & 0.30 & 1.50 & 0.31 & 1.55 \\
Gallium & Ga 69 & 0.03 & 0.15 & 0.11 & 0.53 \\
Germanium & Ge 70 & 0.06 & 0.32 & 0.26 & 1.30 \\
Arsenic & As 75 & 0.19 & 0.97 & 0.82 & 4.11 \\
Selenium & Se 82 & 0.49 & 2.47 & 2.95 & 14.74 \\
Rubidium & Rb 85 & 0.07 & 0.34 & 0.10 & 0.51 \\
Yttrium & Y 89 & 0.16 & 0.79 & 0.06 & 0.29 \\
Zirconium & Zr 90 & 0.01 & 0.07 & 0.06 & 0.31 \\
Niobium & Nb 93 & 0.03 & 0.16 & 0.05 & 0.24 \\
Molybdenum & Mo 95 & 0.04 & 0.21 & 0.20 & 0.99 \\
Ruthenium & Ru 102 & 0.02 & 0.12 & 0.08 & 0.40 \\
\hline
\end{tabular}


Table 2 (continued)

\begin{tabular}{|c|c|c|c|c|c|}
\hline Element & Isotope & Detection I & imits PQ2 & Detection I & imits PQS \\
\hline & & $\mathrm{ppb}(\mathrm{ng} / \mathrm{ml})$ & $\mathrm{ppm}(\boldsymbol{\mu g} / \mathbf{g})$ & $\mathrm{ppb}(\mathrm{ng} / \mathrm{ml})$ & $\mathrm{ppm}(\boldsymbol{\mu g} / \mathbf{g})$ \\
\hline Palladium & Pd 106 & 0.02 & 0.10 & 0.08 & 0.38 \\
\hline Silver & Ag 107 & 0.03 & 0.17 & 0.06 & 0.28 \\
\hline Cadmium & Cd 114 & 0.02 & 0.12 & 0.12 & 0.61 \\
\hline Indium & In 115 & 0.01 & 0.05 & 0.06 & 0.29 \\
\hline Tin & Sn 118 & 0.03 & 0.16 & 0.09 & 0.45 \\
\hline Antimony & Sb 121 & 0.01 & 0.07 & 0.06 & 0.28 \\
\hline Tellurium & Te 126 & 0.13 & 0.64 & 0.29 & 1.45 \\
\hline Cesium & Cs 133 & 0.06 & 0.32 & 0.05 & 0.24 \\
\hline Barium & Ba 138 & 0.02 & 0.10 & 0.02 & 0.10 \\
\hline Lanthanum & La 139 & 0.00 & 0.02 & 0.05 & 0.25 \\
\hline Cerium & Ce 140 & 0.00 & 0.02 & 0.04 & 0.21 \\
\hline Praseodymium & Pr 141 & 0.01 & 0.03 & 0.03 & 0.17 \\
\hline Neodymium & Nd 146 & 0.04 & 0.18 & 0.08 & 0.40 \\
\hline Samarium & Sm 147 & 0.04 & 0.18 & 0.11 & 0.57 \\
\hline Europium & Eu 151 & 0.01 & 0.05 & 0.05 & 0.24 \\
\hline Gadolinium & Gd 157 & 0.03 & 0.15 & 0.10 & 0.50 \\
\hline Terbium & Tb 159 & 0.01 & 0.03 & 0.04 & 0.18 \\
\hline Dysprosium & Dy 162 & 0.03 & 0.13 & 0.06 & 0.28 \\
\hline Holmium & Ho 165 & 0.01 & 0.04 & 0.05 & 0.24 \\
\hline Erbium & Er 166 & 0.02 & 0.09 & 0.06 & 0.29 \\
\hline Ytterbium & Yb 174 & 0.02 & 0.12 & 0.06 & 0.29 \\
\hline Lutetium & Lu 175 & 0.01 & 0.04 & 0.04 & 0.22 \\
\hline Hafnium & Hf 178 & 0.27 & 1.37 & 0.08 & 0.39 \\
\hline Tantalum & Ta 181 & 0.02 & 0.11 & 0.04 & 0.19 \\
\hline Tungsten & W 182 & 0.10 & 0.50 & 0.10 & 0.50 \\
\hline Rhenium & $\operatorname{Re} 185$ & 0.12 & 0.61 & 0.16 & 0.81 \\
\hline Iridium & Ir 193 & 0.04 & 0.19 & 0.08 & 0.39 \\
\hline Platinum & Pt 195 & 0.06 & 0.30 & 0.07 & 0.34 \\
\hline Gold & Au 197 & 0.07 & 0.34 & 0.14 & 0.68 \\
\hline Mercury & Hg 200 & 0.43 & 2.15 & 0.42 & 2.10 \\
\hline Thallium & Tl 205 & 0.04 & 0.20 & 0.04 & 0.20 \\
\hline Lead & $\mathrm{Pb} 208$ & 0.04 & 0.21 & 0.13 & 0.63 \\
\hline Bismuth & Bi 209 & 0.03 & 0.15 & 0.05 & 0.27 \\
\hline Thorium & Th 232 & 0.03 & 0.14 & 0.05 & 0.23 \\
\hline Uranium & U 238 & 0.16 & 0.80 & 0.16 & 0.80 \\
\hline
\end{tabular}

Another method for determining the detection limit in the sample is to determine a method detection limit (MDL) and use it instead of the IDL. In our case the MDL would be determined exactly as the IDL except known levels of very pure plutonium would be added to the blank solutions. As discussed in section IV, the instrument response does decrease with increasing plutonium matrix, thus the MDL increases with increasing plutonium matrix. As shown in Table 3 the detection limits at the instrument are lower in more dilute samples, and for samples below $500 \mu \mathrm{g} \mathrm{Pu} / \mathrm{ml}$ there is no detrimental effect on the instrument detection limit. For this reason, we use the IDL times the dilution factor to calculate analyte detection limits in the sample. 
Table 3. ICP-MS Detection Limits (ppb) in Plutonium

Solutions as a Function of Plutonium Concentration

\begin{tabular}{|l|l|l|l|}
\hline \multicolumn{1}{|c|}{ Isotope } & $\mathbf{2 \%} \mathbf{H N O}_{\mathbf{3}}$ & $\mathbf{5 0 0} \mathbf{p p m} \mathbf{P u}$ & \multicolumn{1}{|c|}{$\mathbf{1 0 0 0} \mathbf{~ p p m ~ P u}$} \\
\hline Be 9 & 0.39 & 0.42 & 0.64 \\
\hline B 11 & 4.3 & 5.6 & 100 \\
\hline Mg 24 & 1.4 & 3.3 & 32 \\
\hline P 31 & 8.1 & 8.9 & 35 \\
\hline Ti 48 & 1.6 & 0.38 & 0.64 \\
\hline V 51 & 0.84 & 1.0 & 8.6 \\
\hline Cr 52 & 0.36 & 0.27 & 1.2 \\
\hline Mn 55 & 0.28 & 0.28 & 0.89 \\
\hline Fe 56 & 16 & 23 & 78 \\
\hline Co 59 & 0.32 & 0.22 & 0.56 \\
\hline Ni 60 & 7.1 & 4.4 & 180 \\
\hline Zn 64 & 6.5 & 5.3 & 120 \\
\hline Cu 65 & 7.4 & 3.1 & 7.4 \\
\hline Ga 69 & 0.29 & 1.1 & 1.0 \\
\hline Sr 88 & 0.20 & 0.26 & 0.42 \\
\hline Y 89 & 0.23 & 0.22 & 0.27 \\
\hline Zr 90 & 0.27 & 0.26 & 0.35 \\
\hline Mo 95 & 0.24 & 0.23 & 0.43 \\
\hline In 115 & 0.13 & 0.39 & 0.35 \\
\hline Sn 124 & 0.14 & 0.31 & 0.59 \\
\hline Ba 138 & 1.0 & 1.2 & 4.4 \\
\hline La 139 & 0.23 & 0.62 & 0.66 \\
\hline Gd 157 & 0.35 & 0.33 & 0.34 \\
\hline Hf 178 & 0.20 & 0.28 & 0.39 \\
\hline Ta 181 & 2.3 & 0.59 & 1.3 \\
\hline W 182 & 2.7 & 3.0 & 6.5 \\
\hline T1 205 & 0.45 & 0.73 & 0.88 \\
\hline Pb 208 & 0.66 & 2.6 & 10 \\
\hline Bi 209 & 0.20 & 0.22 & 0.26 \\
\hline Th 232 & 0.46 & 0.69 & 1.6 \\
\hline U 238 & 0.68 & 5.2 & 29 \\
\hline & & & \\
\hline
\end{tabular}

\section{Phosphorus Analysis}

A number of chemical analysis techniques that were once available to the analytical chemistry group and of benefit to our customers are no longer in service due to the production of mixed hazardous waste. One such technique was the spectrophotometric determination of phosphorous (P). The quantification of $\mathrm{P}$ in 238 plutonium oxide samples is required on a routine basis at a detection limit of $10 \mu \mathrm{g} / \mathrm{g}$ in the solid sample. The atomic emission techniques (ICP-AES and DC-arc AES) cannot meet these requirements.

In order to meet our customers needs, a method using ICP-MS was developed. Phosphorus is mono-isotopic with an amu of 31. Also at 31 amu are isobaric interferences from NO and NOH. Table 4 shows the effect different solutions have on the $\mathrm{NO}$ and $\mathrm{NOH}$ interferences. This data was collected on a high resolution, magnetic sector ICP-MS that is capable of resolving out the individual peaks for $\mathrm{P}, \mathrm{NO}$, and $\mathrm{NOH}$. Even with a dry 
plasma, i.e., a plasma that has no solution running through it, there are $\mathrm{NO}$ and $\mathrm{NOH}$ interferences resulting from the entrainment of atmospheric oxygen and nitrogen. The NO and $\mathrm{NOH}$ interferences increase with the addition of water to the plasma. Hydrochloric acid does not add to the interference level resulting from water, but the nitric acid does. We have also noted that the level of nitrogen and oxygen entrainment is very sensitive to the torch position and small leaks anywhere in the sample introduction system.

Table 4. Integrated Counts for the P, NO, and NOH Peaks as a Function of Solution Type Introduced into the Plasma

\begin{tabular}{|c|c|c|c|}
\hline \multicolumn{4}{|c|}{ Integrated Counts } \\
\hline & $\mathbf{P}$ & NO & NOH \\
\hline $\mathbf{m} / \mathbf{z}$ & 30.974 & 30.995 & 31.006 \\
\hline dry plasma & 7 & 1642 & 315 \\
\hline pure $\mathrm{H}_{2} \mathrm{O}$ & 307 & 14252 & 10024 \\
\hline $1 \% \mathrm{HCl}$ & 704 & 15264 & 12250 \\
\hline $2 \% \mathrm{HCl}$ & 382 & 14334 & 10719 \\
\hline $1 \% \mathrm{HNO}_{3}$ & 192 & 47312 & 35339 \\
\hline $2 \% \mathrm{HNO}_{3}$ & 680 & 75966 & 61237 \\
\hline $10 \mathrm{ppb} \mathrm{P} / \mathrm{H}_{2} \mathrm{O}$ & 1842 & 15043 & 11075 \\
\hline $10 \mathrm{ppb} \mathrm{P} / 1 \% \mathrm{HCl}$ & 12656 & 16286 & 12092 \\
\hline $10 \mathrm{ppb} \mathrm{P} / 1 \% \mathrm{HNO}_{3}$ & 12579 & 45860 & 35474 \\
\hline
\end{tabular}

This data was collected on a Micromass PlasmaTrace 2 high resolution ICP-MS instrument.

A majority of the analytical method is consistent with that described in section IV. The typical digested sample size received is $10 \mathrm{mg} / 2 \mathrm{~mL}$. The original sample is diluted to a final volume of $3 \mathrm{~mL}$ with $1 \% \mathrm{HNO}_{3}$. Because of the $\mathrm{NO} / \mathrm{NOH}$ interference described above, the nitric acid concentration in all the prepared solutions in an analytical run is $1 \%$ $\mathrm{HNO}_{3}$. The internal standards used in the analysis of 238 plutonium oxide samples are Sc and $\mathrm{Bi}$. Scandium is used as an internal standard to monitor and correct for drift of the low mass analytes and bismuth is used as the internal standard to monitor and correct for drift of the high mass analytes. In the past, two dilutions from each sample were analyzed (1:10 and 1:100) for the reasons discussed in section IV. More recently we have been able to eliminate the second dilution by monitoring trends in the data collected for this customer.

\section{Future Improvements}

This paper describes the basis of the ICP-MS analysis method that is routinely used for a wide variety of plutonium samples. We continue to improve the method in several ways. Data is collected and monitored over the long term to understand its long-term precision and accuracy. The addition of new analytes or methods to lower detection limits for analytes requested by our customers are constantly pursued. Waste minimization is important and therefore efforts continue to reduce the waste generated from the analysis. A laser ablation accessory has been installed to perform Laser Ablation ICP-MS. ${ }^{6}$ The data reduction and reporting are a large part of the labor required for ICP-MS analysis. A 
$\mathrm{C}++$ program is being developed to reduce this labor. A high-resolution sector ICP-MS is being installed in a glovebox at the plutonium process facility.

\section{References}

1. M. A. Vaughan and G. Horlick, Applied Spectroscopy 40, 434-445 (1986).

2. S. H. Tan and G. Horlick, Applied Spectroscopy 40, 445-460 (1986).

3. G. R. Gillson, D. J. Douglas, J. E. Fulford, K. W. Halliga, and S. D. Tanner, "Nonspectroscopic Interelement Interferences in Inductively Coupled Plasma Mass Spectrometry," Anal. Chem. 60, 1472 (1988).

4. S. H. Tan and G. Horlick, "Matrix-Effect Observations in Inductively Coupled Plasma Mass Spectrometry," J. Anal. Atom. Spectrom. 2, 745 (1987).

5. B. S. Ross, D. Chambers, and G. Hieftje, "Fundamental and Applied Investigations in Plasma-Source Mass Spectrometry for Elemental Analysis," Mikrochim. Acta, II, (1991).

6. D. J. Figg and L. Drake, "LA-ICP-MS Installed for Plutonium Analysis," Amer. Nucl. Soc., Proceeding from Global '99, 497 (1999). 
This report has been reproduced directly from the best available copy. It is available electronically on the Web (http://www.doe.gov/bridge).

Copies are available for sale to U.S. Department of Energy employees and contractors from-

Office of Scientific and Technical Information

P.O. Box 62

Oak Ridge, TN 37831

(423) 576-8401

Copies are available for sale to the public from-

National Technical Information Service

U.S. Department of Commerce

5285 Port Royal Road

Springfield, VA 22616

(800) 553-6847 


\section{Los Alamos \\ NATIONAL LABORATORY \\ Los Alamos, New Mexico 87545}

